\title{
Extension and lifting of weakly continuous polynomials
}

by

\section{Raffaella Cilia (Catania) and Joaquín M. GutiérRez (Madrid)}

\begin{abstract}
We show that a Banach space $X$ is an $\mathcal{L}_{1}$-space (respectively, an $\mathcal{L}_{\infty^{-}}$ space) if and only if it has the lifting (respectively, the extension) property for polynomials which are weakly continuous on bounded sets. We also prove that $X$ is an $\mathcal{L}_{1}$-space if and only if the space $\mathcal{P}_{\mathrm{wb}}\left({ }^{m} X\right)$ of $m$-homogeneous scalar-valued polynomials on $X$ which are weakly continuous on bounded sets is an $\mathcal{L}_{\infty}$-space.
\end{abstract}

The problem of lifting holomorphic mappings has attracted attention of various authors (see, for instance, [K, A, AMP, GG4]). The extension of holomorphic mappings from a space to a superspace has been treated in many papers (see, for instance, [AB, A, Z, LRy, GG4]). Here we study the extension and lifting of polynomials which are weakly continuous on bounded sets.

This class of polynomials (the definitions will be recalled below) was introduced in $[\mathrm{AP}]$ and has been studied by many authors. In [AHV] it was shown that, if a polynomial is weakly continuous on bounded sets, then it is weakly uniformly continuous on bounded sets. If a dual Banach space $X^{*}$ has the approximation property, then these polynomials on $X$ coincide with the approximable ones [AP, Proposition 2.7]. It was proved in [GG1] that a polynomial $P$ is weakly continuous on bounded sets if and only if it may be factored in the form $P=Q \circ T$, where $T$ is a compact (linear) operator and $Q$ is a polynomial.

We recall the following well known linear results:

TheOREm 1 ([LR, Theorem 4.1]; see also [L]). Let X be a Banach space. Then the following facts are equivalent:

2000 Mathematics Subject Classification: Primary 46G25; Secondary 46B20, 47H60.

Key words and phrases: extension of weakly continuous polynomials, lifting of weakly continuous polynomials, $\mathcal{L}_{1}$-space, $\mathcal{L}_{\infty}$-space.

The first named author was supported by G.N.A.M.P.A. (Italy).

The second named author was supported in part by Dirección General de Investigación, BFM 2003-06420 (Spain). 
(a) $X$ is an $\mathcal{L}_{\infty}$-space;

(b) for all Banach spaces $Z \supseteq Y$, every compact operator $T: Y \rightarrow X$ has an extension to a compact operator $\widetilde{T}: Z \rightarrow X$;

(c) same as in (b) without the requirement of compactness of $\widetilde{T}$;

(d) for all Banach spaces $Z$ and $Y$ with $Z \supseteq X$, every compact operator $T: X \rightarrow Y$ has an extension to a compact operator $\widetilde{T}: Z \rightarrow Y$;

(e) same as in (d) without the requirement of compactness of $\widetilde{T}$.

Let $X, Y$, and $Z$ be Banach spaces and let $\phi: Z \rightarrow Y$ be a surjective operator. A (linear bounded) operator $T: X \rightarrow Y$ is said to admit a lifting to $Z$ (with respect to $\phi$ ) if there is an operator $\widetilde{T}: X \rightarrow Z$ such that $\phi \circ \widetilde{T}=T$. Such a $\widetilde{T}$ is called a lifting of $T$ to $Z$.

Theorem 2 ([LR, Theorem 4.2]). Let $X$ be a Banach space. Then the following facts are equivalent:

(a) $X$ is an $\mathcal{L}_{1}$-space;

(b) for all Banach spaces $Z$ and $Y$ and any surjective operator $\phi$ : $Z \rightarrow Y$, every compact operator $T: X \rightarrow Y$ has a compact lifting $\widetilde{T}: X \rightarrow Z$ with respect to $\phi$

(c) same as in (b) without the requirement of compactness of $\widetilde{T}$;

(d) for all Banach spaces $Z$ and $Y$ and any surjective operator $\phi$ : $Z \rightarrow X$, every compact operator $T: Y \rightarrow X$ has a compact lifting $\widetilde{T}: Y \rightarrow Z$ with respect to $\phi$;

(e) same as in (d) without the requirement of compactness of $\widetilde{T}$.

Throughout, $X, Y$, and $Z$ denote Banach spaces, $X^{*}$ is the dual of $X$, and $B_{X}$ stands for its closed unit ball. The closed unit ball $B_{X^{*}}$ is a compact space when it is endowed with the weak-star topology, which we denote by $w^{*}$. By $\mathbb{N}$ we represent the set of all natural numbers while $\mathbb{K}$ denotes the scalar field. By $X \cong Y$ (respectively, $X \equiv Y$ ), we mean that $X$ and $Y$ are isomorphic (respectively, isometrically isomorphic).

Given $m \in \mathbb{N}$, we denote by $\mathcal{P}\left({ }^{m} X, Y\right)$ the space of all $m$-homogeneous (continuous) polynomials from $X$ into $Y$ endowed with the supremum norm. Recall that to each $P \in \mathcal{P}\left({ }^{m} X, Y\right)$ we can associate a unique symmetric $m$-linear (continuous) mapping $\widehat{P}: X \times \stackrel{(m)}{.} \times X \rightarrow Y$ so that

$$
P(x)=\widehat{P}(x, \stackrel{(m)}{.}, x) \quad(x \in X) .
$$

For simplicity, we write $\mathcal{P}\left({ }^{m} X\right):=\mathcal{P}\left({ }^{m} X, \mathbb{K}\right)$.

For the general theory of multilinear mappings and polynomials on Banach spaces, we refer to [Di2] and [Mu].

We use the notation $\otimes^{m} X:=X \otimes \stackrel{(m)}{*} \otimes X$ for the $m$-fold tensor product of $X$, and $\bigotimes_{\pi}^{m} X$ (respectively, $\bigotimes_{\varepsilon}^{m} X$ ) for the $m$-fold tensor product of $X$ 
endowed with the projective (respectively, injective) norm. Their completions are denoted by $\widetilde{\otimes}_{\pi}^{m} X$ and $\widetilde{\otimes}_{\varepsilon}^{m} X$, respectively (see [DF] or [DU] for the theory of tensor products). By $\otimes_{s}^{m} X:=X \otimes_{s} \stackrel{(m)}{m} \otimes_{s} X$ we denote the $m$-fold symmetric tensor product of $X$, that is, the set of all elements $u \in \bigotimes^{m} X$ of the form

$$
u=\sum_{j=1}^{n} \lambda_{j} x_{j} \otimes \stackrel{(m)}{\cdots} \otimes x_{j} \quad\left(n \in \mathbb{N}, \lambda_{j} \in \mathbb{K}, x_{j} \in X, 1 \leq j \leq n\right) .
$$

The notation $\widetilde{\otimes}_{\pi, s}^{m} X$ stands for the closure of $\bigotimes_{s}^{m} X$ in $\widetilde{\bigotimes}_{\pi}^{m} X$. Given $x_{1}, \ldots, x_{m} \in X$, we define

$$
x_{1} \otimes_{s} \cdots \otimes_{s} x_{m}:=\frac{1}{m !} \sum_{\sigma \in \mathbb{P}_{m}} x_{\sigma(1)} \otimes \cdots \otimes x_{\sigma(m)},
$$

where $\mathbb{P}_{m}$ is the group of all permutations of $m$ elements. For symmetric tensor products, we refer to $[\mathrm{Fl}]$. For simplicity, we shall use the notation

$$
\otimes^{m} x:=x \otimes \stackrel{(m)}{m} \otimes x .
$$

It is well known that $\mathcal{P}\left({ }^{m} X\right) \cong\left(\widetilde{\otimes}_{\pi, s}^{m} X\right)^{*}$ [Fl, Proposition 2.2].

On $\bigotimes_{s}^{m} X$ we define the injective symmetric tensor norm $\varepsilon_{s}$ by $\varepsilon_{s}(z)=\sup \left\{\left|\left\langle z, \otimes^{m} x^{*}\right\rangle\right|: x^{*} \in B_{X^{*}}\right\}=\sup \left\{\left|\sum_{k=1}^{n} \lambda_{k}\left\langle x_{k}, x^{*}\right\rangle^{m}\right|: x^{*} \in B_{X^{*}}\right\}$ for $z=\sum_{k=1}^{n} \lambda_{k} \bigotimes^{m} x_{k}$. We denote by $\bigotimes_{\varepsilon_{s}, s}^{m} X$ the space $\bigotimes_{s}^{m} X$ endowed with the $\varepsilon_{s}$-norm, and by $\widetilde{\bigotimes}_{\varepsilon_{s}, s}^{m} X$ its completion.

If $P \in \mathcal{P}\left({ }^{m} X, Y\right)$, we define its linearization $\bar{P}: \widetilde{\otimes}_{\pi, s}^{m} X \rightarrow Y$ by

$$
\bar{P}\left(\sum_{i=1}^{n} \lambda_{i} x_{i} \otimes \stackrel{(m)}{\cdots} \otimes x_{i}\right)=\sum_{i=1}^{n} \lambda_{i} P\left(x_{i}\right)
$$

for all $\lambda_{i} \in \mathbb{K}, x_{i} \in X(1 \leq i \leq n)$.

If $P \in \mathcal{P}\left({ }^{m} X, Y\right)$, the adjoint of $P$ is the operator $P^{*}: Y^{*} \rightarrow \mathcal{P}\left({ }^{m} X\right)$ given by

$$
P^{*}\left(y^{*}\right)(x)=\left\langle y^{*}, P(x)\right\rangle .
$$

A polynomial $P \in \mathcal{P}\left({ }^{m} X, Y\right)$ is of finite type if it is a finite sum of terms of the form $\gamma^{m} \otimes y$, with $\gamma \in X^{*}$ and $y \in Y$, where $\left(\gamma^{m} \otimes y\right)(x):=\gamma(x)^{m} y$ for all $x \in X$. A polynomial is approximable if it lies in the norm closure of the space of polynomials of finite type. By $\mathcal{P}_{\mathrm{A}}\left({ }^{m} X, Y\right)$ we denote the space of all $m$-homogeneous approximable polynomials from $X$ into $Y$, endowed with the supremum norm.

It is shown in [Fl, Proposition 3.2(6)] that

$$
\mathcal{P}_{\mathrm{A}}\left({ }^{m} X\right) \equiv \widetilde{\bigotimes}_{\varepsilon_{s}, s}^{m} X^{*} .
$$


We are indebted to the referee for pointing out that

$$
\mathcal{P}_{\mathrm{A}}\left({ }^{m} X, Y\right) \equiv \widetilde{\otimes}_{\varepsilon_{s}, s}^{m} X^{*} \widetilde{\otimes}_{\varepsilon} Y .
$$

Indeed, define the operator

$$
J: \mathcal{P}_{\mathrm{A}}\left({ }^{m} X\right) \otimes_{\varepsilon} Y \rightarrow \mathcal{P}\left({ }^{m} X, Y\right)
$$

by

$$
J\left(\sum_{i=1}^{n} P_{i} \otimes y_{i}\right)=Q \quad \text { where } \quad Q(x):=\sum_{i=1}^{n} P_{i}(x) y_{i} \quad(x \in X) .
$$

Then

$$
\begin{aligned}
\|Q\| & =\sup \left\{\|Q(x)\|: x \in B_{X}\right\}=\sup \left\{\left|\left\langle y^{*}, Q(x)\right\rangle\right|: x \in B_{X}, y^{*} \in B_{Y^{*}}\right\} \\
& =\sup \left\{\left|\sum_{i=1}^{n} P_{i}(x) y^{*}\left(y_{i}\right)\right|: x \in B_{X}, y^{*} \in B_{Y^{*}}\right\} .
\end{aligned}
$$

We can embed $\otimes^{m} X$ into $\mathcal{P}_{\mathrm{A}}\left({ }^{m} X\right)^{*}$ by the duality

$$
\left\langle\sum_{i=1}^{n} \lambda_{i} \otimes^{m} x_{i}, P\right\rangle=\sum_{i=1}^{n} \lambda_{i} P\left(x_{i}\right)
$$

It follows that the set $\left\{\otimes^{m} x: x \in B_{X}\right\}$ is norming in the unit ball of $\mathcal{P}_{\mathrm{A}}\left({ }^{m} X\right)^{*}$, so

$$
\begin{aligned}
\|Q\| & =\sup \left\{\left|\sum_{i=1}^{n} \Phi\left(P_{i}\right) y^{*}\left(y_{i}\right)\right|: \Phi \in \mathcal{P}_{\mathrm{A}}\left({ }^{m} X\right)^{*},\|\Phi\| \leq 1, y^{*} \in B_{Y^{*}}\right\} \\
& =\left\|\sum_{i=1}^{n} P_{i} \otimes y_{i}\right\|_{\varepsilon}
\end{aligned}
$$

(see the argument in [Ry, p. 46]) and $J$ is an into isometry. Clearly, the space $\mathcal{P}_{\mathrm{A}}\left({ }^{m} X, Y\right)$ is the range of the extension of $J$ to $\mathcal{P}_{\mathrm{A}}\left({ }^{m} X\right) \widetilde{\otimes}_{\varepsilon} Y$.

A polynomial $P \in \mathcal{P}\left({ }^{m} X, Y\right)$ is compact if $P\left(B_{X}\right)$ is relatively compact in $Y$. A polynomial $P \in \mathcal{P}\left({ }^{m} X, Y\right)$ is compact if and only if its adjoint $P^{*}: Y^{*} \rightarrow \mathcal{P}\left({ }^{m} X\right)$ is a compact operator [AS, Proposition 3.2]. A polynomial $P \in \mathcal{P}\left({ }^{m} X, Y\right)$ is weakly continuous on bounded subsets if for each bounded net $\left(x_{\alpha}\right) \subset X$ weakly converging to $x,\left(P\left(x_{\alpha}\right)\right)$ converges to $P(x)$ in norm. We denote by $\mathcal{P}_{\mathrm{wb}}\left({ }^{m} X, Y\right)$ the space of all polynomials in $\mathcal{P}\left({ }^{m} X, Y\right)$ which are weakly continuous on bounded sets. Every polynomial in $\mathcal{P}_{\mathrm{wb}}\left({ }^{m} X, Y\right)$ is compact ([AP, Lemma 2.2] and [AHV, Theorem 2.9]). An operator is weakly continuous on bounded sets if and only if it is compact [AP, Proposition 2.5]. With each polynomial $P \in \mathcal{P}\left({ }^{m} X, Y\right)$ we associate an operator $T_{P}: X \rightarrow$ $\mathcal{P}\left({ }^{m-1} X, Y\right)$ given by

$$
T_{P}(x)(y):=\widehat{P}(x, y, \stackrel{(m-1)}{\cdots}, y) \quad(x, y \in X) .
$$

Then $P \in \mathcal{P}_{\mathrm{wb}}\left({ }^{m} X, Y\right)$ if and only if $T_{P}$ is compact [AHV, Theorem 2.9]. 
The Banach-Mazur distance $d(X, Y)$ between two isomorphic Banach spaces $X$ and $Y$ is defined by $\inf \left(\|T\|\left\|T^{-1}\right\|\right)$ where the infimum is taken over all isomorphisms $T$ from $X$ onto $Y$. Recall that a Banach space $X$ is an $\mathcal{L}_{p^{-}}$ space $(1 \leq p \leq \infty)[\mathrm{LP}]$ if there is $\lambda \geq 1$ such that every finite-dimensional subspace of $X$ is contained in another subspace $N$ with $d\left(N, \ell_{p}^{n}\right) \leq \lambda$ for some integer $n$.

A Banach space $X$ is an $\mathcal{L}_{p}$-space $(1 \leq p \leq \infty)$ if and only if $X^{*}$ is an $\mathcal{L}_{q}$-space $\left(p^{-1}+q^{-1}=1\right)$ [LR, Theorem III(a)]. An infinite-dimensional complemented subspace of an $\mathcal{L}_{1}$-space (respectively, of an $\mathcal{L}_{\infty}$-space) is an $\mathcal{L}_{1}$-space (respectively, an $\mathcal{L}_{\infty}$-space) [LR, Theorem III(b)].

If $X$ is an $\mathcal{L}_{1}$-space, then, for each $m, \widetilde{\bigotimes}_{\pi}^{m} X$ is an $\mathcal{L}_{1 \text {-space [DF, The- }}$ orem 34.9] (an easy proof may be found in [GG3, Proposition 2.2]). Since $\widetilde{\otimes}_{\pi, s}^{m} X$ is isomorphic to a complemented subspace of $\widetilde{\bigotimes}_{\pi}^{m} X[\mathrm{Fl}$, Proposition 2.3], it follows that $\widetilde{\bigotimes}_{\pi, s}^{m} X$ is an $\mathcal{L}_{1}$-space. Therefore, $\mathcal{P}\left({ }^{m} X\right) \cong$ $\left(\widetilde{\bigotimes}_{\pi, s}^{m} X\right)^{*}$ is an $\mathcal{L}_{\infty}$-space.

If $X$ is an infinite-dimensional $\mathcal{L}_{1}$-space, then $X$ contains a complemented copy of $\ell_{1}$ [LP, Proposition 7.3]. Therefore, $\mathcal{P}_{\mathrm{wb}}\left({ }^{m} X\right)$ is not complemented in $\mathcal{P}\left({ }^{m} X\right)$ [GG2, Lemma 5]. So it is in principle unknown if $\mathcal{P}_{\mathrm{wb}}\left({ }^{m} X\right)$ is an $\mathcal{L}_{\infty}$-space. We now prove that the answer to this question is affirmative. This will be useful later on.

THEOREM 3. Let $X$ be a Banach space. The following facts are equivalent:

(a) $X$ is an $\mathcal{L}_{1}$-space;

(b) for each $m \in \mathbb{N}, \mathcal{P}_{\mathrm{wb}}\left({ }^{m} X\right)$ is an $\mathcal{L}_{\infty}$-space;

(c) there exists $m \in \mathbb{N}$ such that $\mathcal{P}_{\mathrm{wb}}\left({ }^{m} X\right)$ is an $\mathcal{L}_{\infty}$-space.

Proof. (a) $\Rightarrow$ (b). Since $X^{*}$ has the approximation property [DF, p. 306], we have

$$
\mathcal{P}_{\mathrm{wb}}\left({ }^{m} X\right)=\mathcal{P}_{\mathrm{A}}\left({ }^{m} X\right) \equiv \widetilde{\bigotimes}_{\varepsilon_{s}, s}^{m} X^{*}
$$

(see [AP, Proposition 2.7] and our introduction). Now, $X^{*}$ is an $\mathcal{L}_{\infty}$-space, so $\widetilde{\otimes}_{\varepsilon}^{m} X^{*}$ is also an $\mathcal{L}_{\infty}$-space (a proof may be found in [GG3, Proposition 2.2]). Then its complemented subspace $\widetilde{\otimes}_{\varepsilon_{s}, s}^{m} X^{*}[\mathrm{Fl}$, Proposition 3.1] is again an $\mathcal{L}_{\infty}$-space.

(b) $\Rightarrow$ (c) is obvious.

(c) $\Rightarrow$ (a). Since $X^{*}$ is complemented in $\mathcal{P}_{\mathrm{wb}}\left({ }^{m} X\right)$ [AS, Proposition 5.3], $X^{*}$ is an $\mathcal{L}_{\infty}$-space as well. Thus $X$ is an $\mathcal{L}_{1}$-space.

We now give two preparatory results. 
Proposition 4. Let $P \in \mathcal{P}\left({ }^{m} X, Y\right)$. Then its adjoint $P^{*}: Y^{*} \rightarrow$ $\mathcal{P}\left({ }^{m} X\right)$ is $w^{*}-\tau_{\mathrm{p}}$-continuous, where $\tau_{\mathrm{p}}$ is the topology of pointwise convergence on $\mathcal{P}\left({ }^{m} X\right)$. Conversely, let $S: Y^{*} \rightarrow \mathcal{P}\left({ }^{m} X\right)$ be a $w^{*}-\tau_{\mathrm{p}}$-continuous operator. Then there is a polynomial $P \in \mathcal{P}\left({ }^{m} X, Y\right)$ such that $S=P^{*}$.

Proof. Given $P \in \mathcal{P}\left({ }^{m} X, Y\right)$, let $\left(y_{\alpha}^{*}\right)$ be a weak-star null net in $Y^{*}$. Then, for every $x \in X$, we have

$$
P^{*}\left(y_{\alpha}^{*}\right)(x)=\left\langle y_{\alpha}^{*}, P(x)\right\rangle \rightarrow 0,
$$

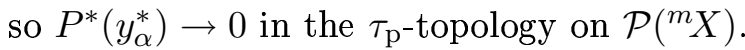

Conversely, let $S: Y^{*} \rightarrow \mathcal{P}\left({ }^{m} X\right)$ be a $w^{*}-\tau_{\mathrm{p}}$-continuous operator. Let

$$
k_{\tilde{\bigotimes}_{\pi, s}^{m} X}: \tilde{\bigotimes}_{\pi, s}^{m} X \rightarrow\left(\tilde{\bigotimes}_{\pi, s}^{m} X\right)^{* *} \cong \mathcal{P}\left({ }^{m} X\right)^{*}
$$

be the natural embedding and let $k_{Y}$ be the natural embedding of $Y$ into $Y^{* *}$. For $x \in X$ fixed, consider the functional $k_{\tilde{\bigotimes}_{\pi, s}^{m} X}\left(\bigotimes^{m} x\right) \circ S: Y^{*} \rightarrow \mathbb{K}$. Clearly,

$$
\left(k_{\tilde{\bigotimes}_{\pi, s}^{m} X}\left(\bigotimes^{m} x\right) \circ S\right)\left(y^{*}\right)=S\left(y^{*}\right)(x) .
$$

By our hypothesis on $S, k_{\tilde{\otimes}_{\pi, s}^{m} X}\left(\bigotimes^{m} x\right) \circ S$ is weak-star continuous on $Y^{*}$ and so it belongs to $k_{Y}(Y)$. Let $P \in \mathcal{P}\left({ }^{m} X, Y\right)$ be defined by $P(x)=$ $k_{Y}^{-1}\left(k_{\tilde{\otimes}_{\pi, s}^{m} X}\left(\bigotimes^{m} x\right) \circ S\right)$. We have

$$
\begin{aligned}
P^{*}\left(y^{*}\right)(x) & =\left\langle y^{*}, P(x)\right\rangle=\left\langle y^{*}, k_{Y}^{-1}\left(k_{\tilde{\Theta}_{\pi, s}^{m} X}\left(\bigotimes^{m} x\right) \circ S\right)\right\rangle \\
& =k_{\widetilde{\Theta}_{\pi, s}^{m} X}\left(\bigotimes^{m} x\right)\left(S\left(y^{*}\right)\right)=S\left(y^{*}\right)(x) .
\end{aligned}
$$

Therefore, $S=P^{*}$.

If $P \in \mathcal{P}_{\mathrm{wb}}\left({ }^{m} X, Y\right)$, then clearly $P^{*}\left(Y^{*}\right) \subseteq \mathcal{P}_{\mathrm{wb}}\left({ }^{m} X\right)$.

Proposition 5. Let $P \in \mathcal{P}_{\mathrm{wb}}\left({ }^{m} X, Y\right)$. Then its adjoint $P^{*}: Y^{*} \rightarrow$ $\mathcal{P}_{\mathrm{wb}}\left({ }^{m} X\right)$ is compact and $w^{*}-\tau_{\mathrm{p}}$-continuous. Conversely, let $S: Y^{*} \rightarrow$ $\mathcal{P}_{\mathrm{wb}}\left({ }^{m} X\right)$ be a compact and $w^{*}-\tau_{\mathrm{p}}$-continuous operator. Then there is $P \in$ $\mathcal{P}_{\mathrm{wb}}\left({ }^{m} X, Y\right)$ such that $S=P^{*}$.

Proof. If $P \in \mathcal{P}_{\mathrm{wb}}\left({ }^{m} X, Y\right)$, then $P^{*}$ is compact and, by Proposition 4 , it is also $w^{*}-\tau_{\mathrm{p}}$-continuous.

Conversely, if $S: Y^{*} \rightarrow \mathcal{P}_{\mathrm{wb}}\left({ }^{m} X\right)$ is a compact and $w^{*}-\tau_{\mathrm{p}}$-continuous operator, then by Proposition 4, there is $P \in \mathcal{P}\left({ }^{m} X, Y\right)$ such that $S=P^{*}$. Since $S$ is compact, $P$ is compact. Since $y^{*} \circ P \in \mathcal{P}_{\mathrm{wb}}\left({ }^{m} X\right)$ for each $y^{*} \in Y^{*}$ it follows from [AP, Proposition 2.8] that $P \in \mathcal{P}_{\mathrm{wb}}\left({ }^{m} X, Y\right)$.

We now extend the equivalences $(\mathrm{a}) \Leftrightarrow(\mathrm{d}) \Leftrightarrow(\mathrm{e})$ of Theorem 2 to the class of polynomials which are weakly continuous on bounded sets.

Theorem 6. Let $X$ be a Banach space. Then the following facts are equivalent: 
(a) $X$ is an $\mathcal{L}_{1}$-space;

(b) for all $m \in \mathbb{N}$, for every surjective operator $\phi: Z \rightarrow X$, every $P \in \mathcal{P}_{\mathrm{wb}}\left({ }^{m} Y, X\right)$ has a lifting $\widetilde{P} \in \mathcal{P}_{\mathrm{wb}}\left({ }^{m} Y, Z\right)$ with respect to $\phi$;

(c) there is $m \in \mathbb{N}$ for which (b) holds;

(d) there is $m \in \mathbb{N}$ for which (b) holds without the requirement that the lifting be weakly continuous on bounded sets.

Proof. (a) $\Rightarrow$ (b). Let $X$ be an $\mathcal{L}_{1}$-space and $P \in \mathcal{P}_{\text {wb }}\left({ }^{m} Y, X\right)$. Then there exist a Banach space $G$, a compact operator $T: Y \rightarrow G$ and a polynomial $Q \in \mathcal{P}\left({ }^{m} G, X\right)$ such that $P=Q \circ T$. Since every compact operator factors through two compact operators [F, Corollary 3.3], we can assume that $Q$ is compact. By [GG4, Theorem 2], there exists a lifting $\widetilde{Q} \in \mathcal{P}\left({ }^{m} G, Z\right)$ with respect to $\phi$. Then $\widetilde{P}:=\widetilde{Q} \circ T \in \mathcal{P}_{\text {wb }}\left({ }^{m} Y, Z\right)$ is a lifting of $P$.

(b) $\Rightarrow$ (c) and (c) $\Rightarrow$ (d) are obvious.

(d) $\Rightarrow$ (a). Let $m \in \mathbb{N}(m \geq 2)$. We show that the statement is true for the index $m-1$. Let $Q \in \mathcal{P}_{\text {wb }}\left({ }^{m-1} Y, X\right)$. By Proposition 5, its adjoint $Q^{*}: X^{*} \rightarrow \mathcal{P}_{\mathrm{wb}}\left({ }^{m-1} Y\right)$ is compact and $w^{*}-\tau_{\mathrm{p}}$-continuous. Choose $e \in Y$ and $\gamma \in Y^{*}$ with $\gamma(e)=1$. Adapting the proof of [AS, Proposition 5.3], we define the operators

$$
j_{Y}: \mathcal{P}_{\mathrm{wb}}\left({ }^{m-1} Y\right) \rightarrow \mathcal{P}_{\mathrm{wb}}\left({ }^{m} Y\right)
$$

by

$$
j_{Y}(R)(y):=\gamma(y) R(y) \quad \text { for } R \in \mathcal{P}_{\mathrm{wb}}\left({ }^{m-1} Y\right), y \in Y,
$$

and

$$
\pi_{Y}: \mathcal{P}_{\mathrm{wb}}\left({ }^{m} Y\right) \rightarrow \mathcal{P}_{\mathrm{wb}}\left({ }^{m-1} Y\right)
$$

given by

$$
\pi_{Y}(S)(y):=\sum_{i=1}^{m}\left(\begin{array}{c}
m \\
i
\end{array}\right)(-1)^{i+1} \gamma(y)^{i-1} \widehat{S}\left(e^{i}, y^{m-i}\right),
$$

where

$$
\widehat{S}\left(e^{i}, y^{m-i}\right):=\widehat{S}\left(e, \stackrel{(i)}{\cdot}, e, y,{ }^{(m-i)}, y\right) .
$$

We show that $\pi_{Y} \circ j_{Y}$ is the identity map on $\mathcal{P}_{\mathrm{wb}}\left({ }^{m-1} Y\right)$ (and, therefore, $j_{Y} \circ \pi_{Y}$ is a projection). Indeed, given $R \in \mathcal{P}_{\mathrm{wb}}\left({ }^{m-1} Y\right)$ and $y \in Y$, since

$$
\widehat{j_{Y}(R)}\left(y_{1}, \ldots, y_{m}\right)=\frac{1}{m} \sum_{i=1}^{m} \gamma\left(y_{i}\right) \widehat{R}\left(y_{1}, \ldots, y_{i-1}, y_{i+1}, \ldots, y_{m}\right),
$$

we have

$$
\begin{aligned}
\pi_{Y}\left(j_{Y}(R)\right)(y)= & \frac{1}{m} \sum_{i=1}^{m}\left(\begin{array}{c}
m \\
i
\end{array}\right)(-1)^{i+1} \gamma(y)^{i-1} \\
& \times\left[i \gamma(e) \widehat{R}\left(e^{i-1}, y^{m-i}\right)+(m-i) \gamma(y) \widehat{R}\left(e^{i}, y^{m-i-1}\right)\right] .
\end{aligned}
$$


For each $i$, we have

$$
\begin{aligned}
\left(\begin{array}{c}
m \\
i-1
\end{array}\right)(-1)^{i}(m-i+1) & \gamma(y)^{i-1} \widehat{R}\left(e^{i-1}, y^{m-i}\right) \\
& +\left(\begin{array}{c}
m \\
i
\end{array}\right)(-1)^{i+1} i \gamma(y)^{i-1} \widehat{R}\left(e^{i-1}, y^{m-i}\right)=0
\end{aligned}
$$

so

$$
\pi_{Y}\left(j_{Y}(R)\right)(y)=R(y) .
$$

The operator $j_{Y} \circ Q^{*}: X^{*} \rightarrow \mathcal{P}_{\mathrm{wb}}\left({ }^{m} Y\right)$ is still compact and $w^{*}-\tau_{\mathrm{p}^{-}}$ continuous. Indeed, if $\left(x_{\alpha}^{*}\right)$ is a net converging to 0 in the weak-star topology of $X^{*}$ and $y \in Y$, we have

$$
j_{Y}\left(Q^{*}\left(x_{\alpha}^{*}\right)\right)(y)=\gamma(y) Q^{*}\left(x_{\alpha}^{*}\right)(y) \rightarrow 0 .
$$

By Proposition 5, there exists a polynomial $P \in \mathcal{P}_{\mathrm{wb}}\left({ }^{m} Y, X\right)$ such that $P^{*}=$ $j_{Y} \circ Q^{*}$. By the hypothesis, there is a lifting $\widetilde{P} \in \mathcal{P}\left({ }^{m} Y, Z\right)$ with respect to $\phi$. Using Proposition 4 and considering $\pi_{Y}$ as an operator $\mathcal{P}\left({ }^{m} Y\right) \rightarrow \mathcal{P}\left({ }^{m-1} Y\right)$, we see that $\pi_{Y} \circ(\widetilde{P})^{*}: Z^{*} \rightarrow \mathcal{P}\left({ }^{m-1} Y\right)$ is $w^{*}-\tau_{\mathrm{p}}$-continuous. So there is $\widetilde{Q} \in \mathcal{P}\left({ }^{m-1} Y, Z\right)$ such that $(\widetilde{Q})^{*}=\pi_{Y} \circ(\widetilde{P})^{*}$. We now prove that $\phi \circ \widetilde{Q}=Q$, equivalently, $(\phi \circ \widetilde{Q})^{*}=Q^{*}$. Indeed,

$$
(\phi \circ \widetilde{Q})^{*}=(\widetilde{Q})^{*} \circ \phi^{*}=\pi_{Y} \circ(\widetilde{P})^{*} \circ \phi^{*}=\pi_{Y} \circ P^{*}=\pi_{Y} \circ j_{Y} \circ Q^{*}=Q^{*} .
$$

So $\widetilde{Q}$ is a lifting of $Q$. Iterating the argument, we deduce that every compact operator from $Y$ into $X$ has a lifting to $Z$. By Theorem $2, X$ is an $\mathcal{L}_{1}$-space.

We now give the equivalences $(\mathrm{a}) \Leftrightarrow(\mathrm{b}) \Leftrightarrow(\mathrm{c})$ of Theorem 2 for polynomials which are weakly continuous on bounded sets.

THEOREM 7. Let $X$ be a Banach space. Then the following facts are equivalent:

(a) $X$ is an $\mathcal{L}_{1}$-space;

(b) for all $m \in \mathbb{N}$, for every surjective operator $\phi: Z \rightarrow Y$, every $P \in$ $\mathcal{P}_{\mathrm{wb}}\left({ }^{m} X, Y\right)$ admits a lifting $\widetilde{P} \in \mathcal{P}_{\mathrm{wb}}\left({ }^{m} X, Z\right)$ with respect to $\phi$;

(c) there exists $m \in \mathbb{N}$ for which (b) holds;

(d) there exists $m \in \mathbb{N}$ for which (b) holds without the requirement that the lifting be weakly continuous on bounded sets.

Proof. $(\mathrm{a}) \Rightarrow(\mathrm{b})$. Let $X$ be an $\mathcal{L}_{1}$-space. Since $X^{*}$ has the approximation property [DF, p. 306], we have

$$
\mathcal{P}_{\mathrm{wb}}\left({ }^{m} X, Y\right) \equiv \mathcal{P}_{\mathrm{A}}\left({ }^{m} X\right) \widetilde{\otimes}_{\varepsilon} Y
$$

(see [AP, Proposition 2.7]). By Theorem 3, $\mathcal{P}_{\mathrm{A}}\left({ }^{m} X\right)=\mathcal{P}_{\mathrm{wb}}\left({ }^{m} X\right)$ is an $\mathcal{L}_{\infty^{-}}$ space. Then the operators

$$
I \otimes \phi: \mathcal{P}_{\mathrm{A}}\left({ }^{m} X\right) \otimes_{\varepsilon} Z \rightarrow \mathcal{P}_{\mathrm{A}}\left({ }^{m} X\right) \otimes_{\varepsilon} Y
$$


and

$$
I \otimes \phi: \mathcal{P}_{\mathrm{A}}\left({ }^{m} X\right) \widetilde{\otimes}_{\varepsilon} Z \rightarrow \mathcal{P}_{\mathrm{A}}\left({ }^{m} X\right) \widetilde{\otimes}_{\varepsilon} Y
$$

are surjective [DF, 23.5, Corollaries 5 and 6], where $I$ is the identity map on $\mathcal{P}_{\mathrm{A}}\left({ }^{m} X\right)$. A standard argument allows us to conclude that every $P \in$ $\mathcal{P}_{\mathrm{wb}}\left({ }^{m} X, Y\right) \equiv \mathcal{P}_{\mathrm{A}}\left({ }^{m} X\right) \widetilde{\otimes}_{\varepsilon} Y$ admits a lifting $\widetilde{P}: \mathcal{P}_{\mathrm{wb}}\left({ }^{m} X, Z\right) \equiv \mathcal{P}_{\mathrm{A}}\left({ }^{m} X\right) \widetilde{\otimes}_{\varepsilon} Z$ with respect to $\phi$.

$(\mathrm{b}) \Rightarrow(\mathrm{c})$ and $(\mathrm{c}) \Rightarrow(\mathrm{d})$ are obvious.

$(d) \Rightarrow(a)$. It is enough to show that the statement is true for the index $m-1$. Define the operators

$$
j_{X}: \mathcal{P}_{\mathrm{wb}}\left({ }^{m-1} X\right) \rightarrow \mathcal{P}_{\mathrm{wb}}\left({ }^{m} X\right), \quad \pi_{X}: \mathcal{P}_{\mathrm{wb}}\left({ }^{m} X\right) \rightarrow \mathcal{P}_{\mathrm{wb}}\left({ }^{m-1} X\right)
$$

as in the proof of Theorem 6 . The proof is analogous to that of $(d) \Rightarrow(a)$ in Theorem 6.

Now we give the equivalences $(\mathrm{a}) \Leftrightarrow(\mathrm{d}) \Leftrightarrow(\mathrm{e})$ of Theorem 1 in the setting of polynomials which are weakly continuous on bounded sets.

THEOREM 8. Let $X$ be a Banach space. Then the following facts are equivalent:

(a) $X$ is an $\mathcal{L}_{\infty}$-space;

(b) for all $m \in \mathbb{N}$, for every into isomorphism $\psi: X \rightarrow Z$, every $P \in$ $\mathcal{P}_{\mathrm{wb}}\left({ }^{m} X, Y\right)$ has an extension $\widetilde{P} \in \mathcal{P}_{\mathrm{wb}}\left({ }^{m} Z, Y\right)$ such that $\widetilde{P} \circ \psi=P$;

(c) there exists $m \in \mathbb{N}$ for which (b) holds;

(d) there exists $m \in \mathbb{N}$ for which (b) holds without the requirement that the extension be weakly continuous on bounded sets.

Proof. $(\mathrm{a}) \Rightarrow(\mathrm{b})$. Let $X$ be an $\mathcal{L}_{\infty}$-space. Let $\psi: X \rightarrow Z$ be an into isomorphism, and let $P \in \mathcal{P}_{\mathrm{wb}}\left({ }^{m} X, Y\right)$. Then there exist a Banach space $G$, a compact operator $T: X \rightarrow G$ and a polynomial $Q \in \mathcal{P}\left({ }^{m} G, Y\right)$ such that $P=Q \circ T$. By Theorem 1 , there is a compact operator $\widetilde{T}: Z \rightarrow G$ such that $\widetilde{T} \circ \psi=T$. Define $\widetilde{P}:=Q \circ \widetilde{T} \in \mathcal{P}\left({ }^{m} Z, Y\right)$. Then $\widetilde{P}$ is an extension of $P$, and $\widetilde{P} \in \mathcal{P}_{\mathrm{wb}}\left({ }^{m} Z, Y\right)$.

$(\mathrm{b}) \Rightarrow(\mathrm{c})$ and $(\mathrm{c}) \Rightarrow(\mathrm{d})$ are obvious.

$(\mathrm{d}) \Rightarrow(\mathrm{a})$. We can assume $m \geq 2$. We prove that, for every into isomorphism $\psi: X \rightarrow Z$, every $Q \in \mathcal{P}_{\mathrm{wb}}\left({ }^{m-1} X, Y\right)$ has an extension $\widetilde{Q} \in$ $\mathcal{P}\left({ }^{m-1} Z, Y\right)$ such that $\widetilde{Q} \circ \psi=Q$. Let $\bar{Q}: \widetilde{\otimes}_{\pi, s}^{m-1} X \rightarrow Y$ be the linearization of $Q$. Choose $x_{0} \in X$ and let $z_{0}:=\psi\left(x_{0}\right)$. Select $z^{*} \in Z^{*}$ with $z^{*}\left(z_{0}\right)=1$ and let $x^{*}=\psi^{*}\left(z^{*}\right)$. Then $x^{*}\left(x_{0}\right)=1$. Let

$$
\Pi_{X}: \widetilde{\bigotimes}_{\pi, s}^{m} X \rightarrow \tilde{\otimes}_{\pi, s}^{m-1} X, \quad J_{X}: \widetilde{\bigotimes}_{\pi, s}^{m-1} X \rightarrow \widetilde{\otimes}_{\pi, s}^{m} X
$$

be the operators defined in [Bl, p. 168] by

$$
\Pi_{X}\left(\bigotimes^{m} x\right)=x^{*}(x) \bigotimes^{m-1} x
$$


and

$J_{X}\left(\otimes^{m-1} x\right)=\sum_{k=1}^{m}\left(\begin{array}{c}m \\ k\end{array}\right)(-1)^{k+1} x^{*}(x)^{k-1} x_{0} \otimes_{s} \stackrel{(k)}{.} \otimes_{s} x_{0} \otimes_{s} x \otimes_{s} \stackrel{(m-k)}{\cdots} \otimes_{s} x$.

It is shown in [Bl, p. 168] that $\Pi_{X} \circ J_{X}$ is the identity map on $\tilde{\otimes}_{\pi, s}^{m-1} X$. Similarly we can define

$$
J_{Z}: \widetilde{\bigotimes}_{\pi, s}^{m-1} Z \rightarrow \widetilde{\bigotimes}_{\pi, s}^{m} Z
$$

by

$$
J_{Z}\left(\otimes^{m-1} z\right)=\sum_{k=1}^{m}\left(\begin{array}{c}
m \\
k
\end{array}\right)(-1)^{k+1} z^{*}(z)^{k-1} z_{0} \otimes_{s} \stackrel{(k)}{.} \otimes_{s} z_{0} \otimes_{s} z \otimes_{s} \stackrel{(m-k)}{\cdots} \otimes_{s} z .
$$

Consider $S:=\bar{Q} \circ \Pi_{X}: \widetilde{\bigotimes}_{\pi, s}^{m} X \rightarrow Y$ and let $P \in \mathcal{P}\left({ }^{m} X, Y\right)$ be the polynomial whose linearization is $S$. Then, for each $x \in X$,

$$
P(x)=S\left(\bigotimes^{m} x\right)=\bar{Q} \circ \Pi_{X}\left(\bigotimes^{m} x\right)=x^{*}(x) \bar{Q}\left(\bigotimes^{m-1} x\right)=x^{*}(x) Q(x) .
$$

Since $Q \in \mathcal{P}_{\mathrm{wb}}\left({ }^{m-1} X, Y\right)$, it follows that $P \in \mathcal{P}_{\mathrm{wb}}\left({ }^{m} X, Y\right)$. By our hypothesis, there is $\widetilde{P} \in \mathcal{P}\left({ }^{m} Z, Y\right)$ such that $\widetilde{P} \circ \psi=P$. Let $\overline{\widetilde{P}}$ be the linearization of $\widetilde{P}$, and consider the composition $\overline{\widetilde{P}} \circ J_{Z}: \widetilde{\otimes}_{\pi, s}^{m-1} Z \rightarrow Y$. Let $\widetilde{Q} \in$ $\left.\mathcal{P}{ }^{m-1} Z, Y\right)$ be the polynomial whose linearization coincides with $\overline{\widetilde{P}} \circ J_{Z}$. We show that $\widetilde{Q}$ extends $Q$, that is, $\widetilde{Q} \circ \psi=Q$, equivalently, $\widetilde{Q} \circ \psi=\bar{Q}$. Indeed,

$$
\begin{aligned}
& \widetilde{\widetilde{Q} \circ \psi}\left(\bigotimes^{m-1} x\right)=\overline{\widetilde{Q}}\left(\bigotimes^{m-1} \psi(x)\right)=\left(\overline{\widetilde{P}} \circ J_{Z}\right)\left(\bigotimes^{m-1} \psi(x)\right) \\
& =\overline{\widetilde{P}}\left[\sum_{k=1}^{m}\left(\begin{array}{c}
m \\
k
\end{array}\right)(-1)^{k+1} z^{*}(\psi(x))^{k-1}\right. \\
& \left.\times \psi\left(x_{0}\right) \otimes_{s} \stackrel{(k)}{.} \otimes_{s} \psi\left(x_{0}\right) \otimes_{s} \psi(x) \otimes_{s} \stackrel{(m-k)}{\cdots} \otimes_{s} \psi(x)\right] \\
& =\sum_{k=1}^{m}\left(\begin{array}{c}
m \\
k
\end{array}\right)(-1)^{k+1} z^{*}(\psi(x))^{k-1} \overline{\widetilde{P} \circ \psi}\left(x_{0} \otimes_{s} \stackrel{(k)}{\cdots} \otimes_{s} x_{0} \otimes_{s} x \otimes_{s} \stackrel{(m-k)}{\cdots} \otimes_{s} x\right) \\
& =\sum_{k=1}^{m}\left(\begin{array}{c}
m \\
k
\end{array}\right)(-1)^{k+1} z^{*}(\psi(x))^{k-1} \bar{P}\left(x_{0} \otimes_{s} \stackrel{(k)}{\cdots} \otimes_{s} x_{0} \otimes_{s} x \otimes_{s} \stackrel{(m-k)}{\cdots} \otimes_{s} x\right) \\
& =\bar{P}\left[\sum_{k=1}^{m}\left(\begin{array}{c}
m \\
k
\end{array}\right)(-1)^{k+1} x^{*}(x)^{k-1} x_{0} \otimes_{s} \stackrel{(k)}{\cdots} \otimes_{s} x_{0} \otimes_{s} x \otimes_{s} \stackrel{(m-k)}{\cdots} \otimes_{s} x\right] \\
& =\bar{P} \circ J_{X}\left(\bigotimes^{m-1} x\right)=\bar{Q} \circ \Pi_{X} \circ J_{X}\left(\bigotimes^{m-1} x\right)=\bar{Q}\left(\bigotimes^{m-1} x\right) .
\end{aligned}
$$

Iterating the argument, we conclude that every compact operator $X \rightarrow Y$ has an extension, and so $X$ is an $\mathcal{L}_{\infty}$-space by Theorem 1 . 
Remark 9. The implications $(\mathrm{a}) \Rightarrow(\mathrm{b})$ and $(\mathrm{a}) \Rightarrow(\mathrm{c})$ of Theorem 1 do not extend to the polynomials which are weakly continuous on bounded sets. We shall see that, for every Banach space $X \neq\{0\}$, there are a polynomial $P \in \mathcal{P}_{\mathrm{wb}}\left({ }^{2} \ell_{p}, X\right)(p \geq 2)$ and a Banach space $Z$ containing $\ell_{p}$ such that $P$ does not admit an extension $\widetilde{P}: Z \rightarrow X$. Indeed, choose $x_{0} \in X$ and $x^{*} \in X^{*}$ with $x^{*}\left(x_{0}\right)=1$; define

$$
P(y)=\left(\sum_{n=1}^{\infty} \frac{1}{n} y_{n}^{2}\right) x_{0} \quad \text { for } y=\left(y_{n}\right)_{n=1}^{\infty} \in \ell_{p}
$$

Then $P$ is approximable, so $P \in \mathcal{P}_{\text {wb }}\left({ }^{2} \ell_{p}, X\right)$.

The polynomial $Q \in \mathcal{P}_{\mathrm{wb}}\left({ }^{2} \ell_{p}\right)(p \geq 2)$ given by

$$
Q(y)=\sum_{n=1}^{\infty} \frac{1}{n} y_{n}^{2}
$$

is not extendible [C, Example 2.6]. Hence there exists a Banach space $Z$ containing $\ell_{p}$ such that $Q$ does not admit an extension to $Z$. Denote by $\psi$ the embedding of $\ell_{p}$ into $Z$. Suppose there is a polynomial $\widetilde{P} \in \mathcal{P}\left({ }^{2} Z, X\right)$ with $\widetilde{P} \circ \psi=P$. Let $N$ be the subspace generated by $x_{0}$, let $\pi: X \rightarrow N$ be the projection given by $\pi(x)=x^{*}(x) x_{0}(x \in X)$, and denote by $i: N \rightarrow \mathbb{K}$ the natural isomorphism. Then $i \circ \pi \circ \widetilde{P} \circ \psi(y)=i(\pi(P(y)))=i\left(\pi\left(\left(\sum_{n=1}^{\infty} \frac{1}{n} y_{n}^{2}\right) x_{0}\right)\right)=\sum_{n=1}^{\infty} \frac{1}{n} y_{n}^{2}=Q(y)$, so $i \circ \pi \circ \widetilde{P}$ is an extension of $Q$, which is impossible.

We shall now prove that there is no "dual" version of Theorem 3. We recall some preliminary definitions.

A Banach space $X$ is finitely representable in a Banach space $Y$ if, for each $\varepsilon>0$ and each finite-dimensional subspace $M$ of $X$, there is a finitedimensional subspace $N$ of $Y$ and a bijective operator $T: M \rightarrow N$ such that $\|T\|\left\|T^{-1}\right\| \leq 1+\varepsilon$.

If $\mathcal{P}$ is a property defined for Banach spaces, that is, $\mathcal{P}$ is a subclass of the class of all Banach spaces, then a Banach space $X$ has property "super $\mathcal{P}$ " if every Banach space finitely representable in $X$ also has $\mathcal{P}$. A property $\mathcal{P}$ is called a superproperty if $\mathcal{P}=\operatorname{super} \mathcal{P}$ (see [Be, Chapter 4.I]).

THEOREM 10. If $X$ is an infinite-dimensional Banach space, then $\mathcal{P}_{\mathrm{wb}}\left({ }^{m} X\right)$ and $\mathcal{P}\left({ }^{m} X\right)$ are not $\mathcal{L}_{p}$-spaces, for $1 \leq p<\infty$ and $m \in \mathbb{N}(m \geq 2)$.

Proof. It is shown in [Di1, Corollary 3] that $\ell_{\infty}$ is finitely representable in $\mathcal{P}\left({ }^{m} X\right)$. In fact, the proof is also valid for $\mathcal{P}_{\mathrm{wb}}\left({ }^{m} X\right)$. Suppose that $\mathcal{P}_{\mathrm{wb}}\left({ }^{m} X\right)$ (respectively, $\mathcal{P}\left({ }^{m} X\right)$ ) is an $\mathcal{L}_{p}$-space for some $1 \leq p<\infty$. Then $\mathcal{P}_{\text {wb }}\left({ }^{m} X\right)$ 
(respectively, $\mathcal{P}\left({ }^{m} X\right)$ ) has finite cotype [DJT, Corollary 11.7(a)], which implies that $\ell_{\infty}$ also has finite cotype [DJT, Theorem 11.6], and this contradicts a well known result [DJT, Corollary 11.7(b)].

The above proof shows that $\mathcal{P}_{\mathrm{wb}}\left({ }^{m} X\right)$ and $\mathcal{P}\left({ }^{m} X\right)$ do not have finite cotype. Since $\ell_{\infty}$ does not have type $>1$ [DJT, Corollary 11.7(b)], neither do $\mathcal{P}_{\mathrm{wb}}\left({ }^{m} X\right)$ and $\mathcal{P}\left({ }^{m} X\right)$. These results are mentioned in [Fl, 3.3].

More generally, from the fact that every Banach space is finitely representable in $\ell_{\infty}$ [FHH, Theorem 9.14], it follows that, if $X$ is an infinitedimensional Banach space, then $\mathcal{P}_{\mathrm{wb}}\left({ }^{m} X\right)$ and $\mathcal{P}\left({ }^{m} X\right)$ do not have any nontrivial superproperty, for $m \in \mathbb{N}(m \geq 2)$. This result is mentioned in the introduction to [Di1].

Clearly, all these results (from Theorem 10 on) are also true for the space $\mathcal{L}\left({ }^{m} X\right)$ of $m$-linear forms $(m \geq 2)$.

We are grateful to the referee for many suggestions that have improved the paper.

\section{References}

[A] R. M. Aron, Extension and lifting theorems for analytic mappings, in: K.-D. Bierstedt and B. Fuchssteiner (eds.), Functional Analysis: Surveys and Recent Results II, North-Holland Math. Stud. 38, North-Holland, Amsterdam, 1980, $257-267$.

[AB] R. M. Aron and P. D. Berner, A Hahn-Banach extension theorem for analytic mappings, Bull. Soc. Math. France 106 (1978), 3-24.

[AHV] R. M. Aron, C. Hervés, and M. Valdivia, Weakly continuous mappings on Banach spaces, J. Funct. Anal. 52 (1983), 189-204.

[AMP] R. M. Aron, L. A. Moraes, and O. W. Paques, Lifting of holomorphic mappings, Proc. Roy. Irish Acad. Sect. A 94 (1994), 119-126.

[AP] R. M. Aron and J. B. Prolla, Polynomial approximation of differentiable functions on Banach spaces, J. Reine Angew. Math. 313 (1980), 195-216.

[AS] R. M. Aron and M. Schottenloher, Compact holomorphic mappings on Banach spaces and the approximation property, J. Funct. Anal. 21 (1976), 7-30.

[Be] B. Beauzamy, Introduction to Banach Spaces and Their Geometry, North-Holland Math. Stud. 68, North-Holland, Amsterdam, 1982.

[Bl] F. Blasco, Complementation in spaces of symmetric tensor products and polynomials, Studia Math. 123 (1997), 165-173.

[C] D. Carando, Extendibility of polynomials and analytic functions on $\ell_{p}$, ibid. 145 (2001), 63-73.

[DF] A. Defant and K. Floret, Tensor Norms and Operator Ideals, North-Holland Math. Stud. 176, North-Holland, Amsterdam, 1993.

[DJT] J. Diestel, H. Jarchow, and A. Tonge, Absolutely Summing Operators, Cambridge Stud. Adv. Math. 43, Cambridge Univ. Press, Cambridge, 1995.

[DU] J. Diestel and J. J. Uhl, Jr., Vector Measures, Math. Surveys Monogr. 15, Amer. Math. Soc., Providence, RI, 1977. 
[Di1] S. Dineen, A Dvoretzky theorem for polynomials, Proc. Amer. Math. Soc. 123 (1995), 2817-2821.

[Di2] -, Complex Analysis on Infinite Dimensional Spaces, Springer Monogr. Math., Springer, Berlin, 1999.

[FHH] M. Fabian, P. Habala, P. Hájek, V. Montesinos, J. Pelant, and V. Zizler, Functional Analysis and Infinite-Dimensional Geometry, CMS Books in Math. 8, Springer, New York, 2001.

[F] T. Figiel, Factorization of compact operators and applications to the approximation problem, Studia Math. 45 (1973), 191-210.

[Fl] K. Floret, Natural norms on symmetric tensor products of normed spaces, Note Mat. 17 (1997), 153-188.

[GG1] M. González and J. M. Gutiérrez, Factorization of weakly continuous holomorphic mappings, Studia Math. 118 (1996), 117-133.

[GG2] -, 一, The polynomial property (V), Arch. Math. (Basel) 75 (2000), 299-306.

[GG3] -, -, Tensor products and dual spaces with the Dunford-Pettis property, Rend. Circ. Mat. Palermo (2) Suppl. 68 (2002), 479-490.

[GG4] -, -, Extension and lifting of polynomials, Arch. Math. (Basel) 81 (2003), $431-438$.

[K] W. Kaballo, Lifting theorems for vector valued functions and the $\varepsilon$-tensor prod$u c t$, in: K.-D. Bierstedt and B. Fuchssteiner (eds.), Functional Analysis: Surveys and Recent Results, North-Holland Math. Stud. 27, North-Holland, Amsterdam, $1977,149-166$.

[L] J. Lindenstrauss, Extensions of compact operators, Mem. Amer. Math. Soc. 48 (1964).

[LP] J. Lindenstrauss and A. Pełczyński, Absolutely summing operators in $\mathcal{L}_{p}$-spaces and their applications, Studia Math. 29 (1968), 275-326.

[LR] J. Lindenstrauss and H. P. Rosenthal, The $\mathcal{L}_{p}$-spaces, Israel J. Math. 7 (1969), 325-349.

[LRy] M. Lindström and R. A. Ryan, Applications of ultraproducts to infinite dimensional holomorphy, Math. Scand. 71 (1992), 229-242.

[Mu J. Mujica, Complex Analysis in Banach Spaces, North-Holland Math. Stud. 120, North-Holland, Amsterdam, 1986.

[Ry] R. A. Ryan, Introduction to Tensor Products of Banach Spaces, Springer Monogr. Math., Springer, Berlin, 2002.

[Z] I. Zalduendo, A canonical extension for analytic functions on Banach spaces, Trans. Amer. Math. Soc. 320 (1990), 747-763.

Dipartimento di Matematica

Facoltà di Scienze

Università di Catania

Viale Andrea Doria 6

95125 Catania, Italy

E-mail: cilia@dmi.unict.it
Departamento de Matemática Aplicada ETS de Ingenieros Industriales Universidad Politécnica de Madrid

C. José Gutiérrez Abascal 2 28006 Madrid, Spain E-mail: jgutierrez@etsii.upm.es

Received April 16, 2004

Revised version December 7, 2004 